\title{
Pulse pressure during the initial resuscitative period in patients with septic shock treated with a protocol-driven resuscitation bundle therapy
}

\author{
Sang-Hun Lee ${ }^{1,}$, Youn-Jung Kim ${ }^{2,}$, Gi Na Yu ${ }^{2, \dagger}$, Jae Cheon Jeon ${ }^{1}$, and Won Young Kim ${ }^{2}$
}

${ }^{1}$ Department of Emergency Medicine, Keimyung University Dongsan Medical Center, Daegu; ${ }^{2}$ Department of Emergency Medicine, Asan Medical Center, University of Ulsan College of Medicine, Seoul, Korea

Received: February 13, 2020

Revised : March 22, 2020

Accepted: April 26, 2020

\section{Correspondence to}

Won Young Kim, M.D.

Department of Emergency

Medicine, Asan Medical Center,

University of Ulsan College of

Medicine, 88 Olympic-ro 43-gil,

Songpa-gu, Seoul 05505, Korea

Tel: $+82-2-3010-3350$

Fax: $+82-2-3010-3360$

E-mail:wonpia73@naver.com https://orcid.org/0000-00026904-5966

*These authors contributed equally to this work.

${ }^{\dagger}$ Current affiliation: Department of Emergency Medicine, University of Yonsei College of Medicine, Seoul, Korea
Background/Aims: Maintaining a mean arterial pressure (MAP) $\geq 65 \mathrm{mmHg}$ during septic shock should be based on individual circumstances, but specific target is poorly understood. We investigated associations between time-weighted average (TWA) hemodynamic parameters during the initial resuscitative period and 28-day mortality.

Methods: Prospectively collected data were obtained from a septic shock patient registry, according to the Sepsis-3 definition, between 2016 and 2018. The TWA systolic blood pressure, diastolic blood pressure, MAP, shock index, and pulse pressure (PP) during the first 6 hours after shock recognition were compared. Multivariable regression analysis was performed to assess associations between these parameters and 28 -day mortality.

Results: Of 340 patients with septic shock, 92 died. Only the median TWA PP differed between the survivors and non-survivors $39.2 \mathrm{mmHg}$ vs. $43.0 \mathrm{mmHg}, p=$ 0.020), whereas the other indexes did not. When PP was divided into quartiles (< 34,34 to 40,40 to 48 , and $>48 \mathrm{mmHg}$ ), the mortality rate was higher in the highest quartile (41.2\%). Multivariable logistic analysis revealed that PP (odds ratio [OR], 1.28; $95 \%$ confidence interval [CI], 1.012 to $1.622 ; p=0.039$ ) and $\mathrm{PP}$ of $>48 \mathrm{mmHg}$ (OR, 2.25; 95\% CI, 1.272 to $3.981 ; p=0.005$ ) were independently associated with 28 day mortality.

Conclusions: PP was significantly associated with 28 -day mortality in patients with septic shock and MAP maintained at $>65 \mathrm{mmHg}$ during the first 6 hours. Further studies are warranted to optimize strategies for maintaining PP and MAP at $>65 \mathrm{mmHg}$ during the early resuscitative period.

Keywords: Septic shock; Blood pressure; Prognosis; Emergencies; Critical care

\section{INTRODUCTION}

Septic shock is a life-threatening state caused by a dysregulated host response to infection [1]. Despite recent medical advances and efforts, septic shock remains one of the global leading causes of death [1]. Early recognition and vigorous resuscitative treatments such as adminis- tration of broad-spectrum antibiotic therapy, adequate fluids, and vasopressors are key factors for improving outcomes in patients with sepsis [2]. The guidelines of the Surviving Sepsis Campaign recommend that mean arterial pressure (MAP) be maintained at $\geq 65 \mathrm{mmHg}$ in patients with septic shock (Grade $1 \mathrm{~B}$ recommendation) [2]. 
MAP reflects the driving pressure of tissue perfusion, and targeting MAPs of $\geq 65 \mathrm{mmHg}$ may restore adequate organ perfusion in patients with septic shock, as their autoregulatory system for maintaining regional perfusion is impaired $[2,3]$. A recent multicenter trial showed no significant differences in mortality rates between high- (8o to $85 \mathrm{mmHg}$ ) and low-target (65 to $70 \mathrm{mmHg}$ ) patients with septic shock; however, high-target patients with a medical history of chronic hypertension had significantly lower requirements for renal replacement therapy [4]. Besides, MAP does not reflect age-related changes in blood pressure as well as pulse pressure (PP) [5]. Therefore, personalized guidelines for optimizing blood pressure management during the initial resuscitative period seem to be necessary to optimize outcomes in patients with septic shock [2].

$\mathrm{PP}$ is defined as the difference between systolic blood pressure (SBP) and diastolic blood pressure (DBP). A high PP may be a sign of stiffness of the arterial walls and has been associated with mortality in patients with cardiovascular disease, those undergoing hemodialysis, and those with ischemic stroke [6-9]. Little is known, however, about the relationship between PP and septic shock. PP may have a distinct pathophysiological mechanism associated with outcomes in patients with septic shock and may provide additional information, especially in patients with septic shock treated by targeting a MAP of $\geq 65 \mathrm{mmHg}$. A better understanding of hemodynamic parameters during the initial resuscitative period in patients with septic shock will contribute to improvements of the initial resuscitative strategy.

We hypothesized PP would have prognostic value for the outcomes of septic shock patients because it is influenced by arterial stiffness, heart function, and intra-vascular volume, which together sensitively reflect the patient's status. The aim of the present study was to investigate the association between time-weighted average (TWA) hemodynamic parameters, including SBP, DBP, MAP, shock index (SI), and PP, during the initial resuscitative period and 28-day mortality in patients with septic shock treated with a protocol-driven resuscitation therapy bundle, including maintaining the MAP at $\geq 65 \mathrm{mmHg}$.

\section{METHODS}

\section{Study design and participants}

We performed a retrospective cohort study based on a single-center, prospectively collected registry. The emergency department (ED) of Asan Medical Center has participated the Korean Shock Society (KoSS) septic shock registry study since October 2015. The KoSS septic shock registry study collects data pertaining to patients with septic shock at 10 teaching hospitals across South Korea [10]. Following the protocol of KoSS septic shock registry study, our center included all adult patients $(\geq$ 19 years) when they showed evidence of refractory hypotension or hypoperfusion with suspected or confirmed infection within 6 hours after ED presentation $[1,11]$. Refractory hypotension was defined as sustained hypotension despite an intravenous fluid supply of $\geq$ 20 to $30 \mathrm{~mL} / \mathrm{kg}$ or the need for vasopressors to preserve an SBP of $>90 \mathrm{mmHg}$ or MAP of $>70 \mathrm{mmHg}$ [11]. Hypoperfusion was defined as a lactate concentration of $>4 \mathrm{mmol} / \mathrm{L}$ [12]. Patients were excluded if they refused intensive management or consented to a "do not resuscitate" order, developed septic shock 6 hours after ED arrival, were directly transferred to another hospital at ED, or refused enrollment in the septic shock registry [13-15]. Among the septic shock patients, about $75 \%$ of patients were finally enrolled. The most common reason for exclusion was having "do not resuscitate" order (56\%), followed by refusal of enrollment (28\%), development of septic shock 6 hours after ED arrival (12\%), and a direct transfer to another hospital at ED (4\%). Patients were followed up until 28 days after hospital admission or the time of death. The study protocol was approved by the Institutional Review Board of Asan Medical Center (IRB No. 2018-1020), and informed consent was obtained prior to patient enrollment.

In this study, we extracted the patients who met the criteria of the Third International Consensus for Sepsis and Septic Shock (Sepsis-3), and admitted to ED between June 1, 2016, and April 30, 2018. The Sepsis-3 criteria include persistent hypotension requiring vasopressors and a serum lactate concentration of $>2 \mathrm{mmol} / \mathrm{L}$ after adequate fluid administration [1]. Patients were categorized into 28-day survivor and non-survivor groups. 


\section{Management and data collection}

All the patients were treated with a protocol-driven resuscitation bundle therapy in accordance with the Surviving Sepsis Campaign guidelines, which included adequate fluid resuscitation (at least $30 \mathrm{mg} / \mathrm{kg}$ of crystalloids), vasopressor administration, acquisition of blood cultures before starting the antimicrobial therapy and antibiotic treatment $[2,11]$. We used norepinephrine (NE) as an initial vasopressor agent and added vasopressin at a dose of 0.04 units/minute when vital signs were unstable despite the use of NE at a dose of $\geq 0.2 \mu \mathrm{g} / \mathrm{kg} / \mathrm{min}$. And we added epinephrine when vital sign was not stabilized, if NE infusion rate of $0.4 \mu \mathrm{g} / \mathrm{kg} / \mathrm{min}$ or more was required. Arterial and central venous lines were routinely inserted and monitored continuously in patients who required high-dose vasopressors, defined as a dosage of $\geq 0.1 \mu \mathrm{g} / \mathrm{kg} / \mathrm{min}$, to achieve MAPs of $\geq 65 \mathrm{mmHg}$.

Information on demographic and clinical characteristics, including age, sex, comorbid diseases, infection focus, laboratory findings, and 28-day mortality were obtained from the registry. We analyzed the vital signs measured during the first 6 hours after the recognition of septic shock. These data were extracted from the electronic medical records. SBP, DBP, and heart rate (HR) were recorded automatically every minute in the intensive care unit and manually at least once every hour in the ED. As manual recordings input into the electronic medical records in the ED may contain errors, SBPs higher than $220 \mathrm{mmHg}$ or those lower than their corresponding DBP (or $20 \mathrm{mmHg}$ ) were discarded because such values are improbable.

TWA hemodynamic parameters were calculated, including SBP, DBP, HR, and MAP. The MAP was calculated as $2 / 3 \times \mathrm{DBP}+1 / 3 \times \mathrm{SBP}$. Because of their unequal intervals of measurements, TWA was used to estimate their averages. TWA parameters were calculated using the following equation (Fig. 1).

$$
\text { TWA value }=\sum_{\iota=1}^{\eta}([T \iota+1-T \iota] \times A \iota) \div \sum_{\iota=1}^{\eta} T \iota
$$

In addition, the TWA of PP (SBP - DBP) and SI (HR ( SBP) were determined. The primary outcome was 28day mortality.

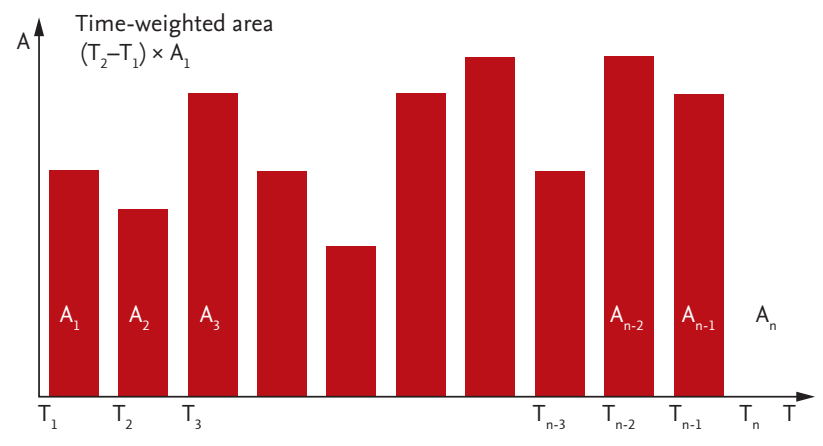

Figure 1. Time-weighted average calculation formula.

\section{Statistical analysis}

Non-normally distributed continuous variables, as determined using Kolmogorov-Smirnov tests, are reported as median and interquartile ranges (IQRs) and compared using Mann-Whitney $U$ tests. Categorical variables are reported as number and percentage and compared using the chi-square or Fisher exact test, as appropriate. The associations of the baseline characteristics and hemodynamic parameters with the 28-day mortality rates were analyzed using univariable logistic regression. The variables with an entry-level significance of $p$ values of $<0.10$ in univariable analysis, age, and sex were selected for multivariable stepwise logistic regression analysis with a backward elimination method. The results were summarized by estimating the adjusted odds ratios (ORs) and 95\% confidence intervals (CIs). Models were tested for goodness-of-fit using the Hosmer-Lemeshow method (Supplementary Table 1). Two-sided $p$ values of $<0.05$ were considered statistically significant. All statistical analyses were performed using IBM SPSS Statistics version 21.0 for Windows (IBM Corp., Armonk, NY, USA).

\section{RESULTS}

\section{Baseline characteristics}

During the study period, 523 patients with septic shock were enrolled in the septic shock registry, among whom 340 fulfilled the clinical criteria for septic shock according to the Sepsis-3 definitions and were included in this study (Fig. 2). The median number of vital signs checks per patient enrolled with septic shock was 10 (IQR, 8 to 


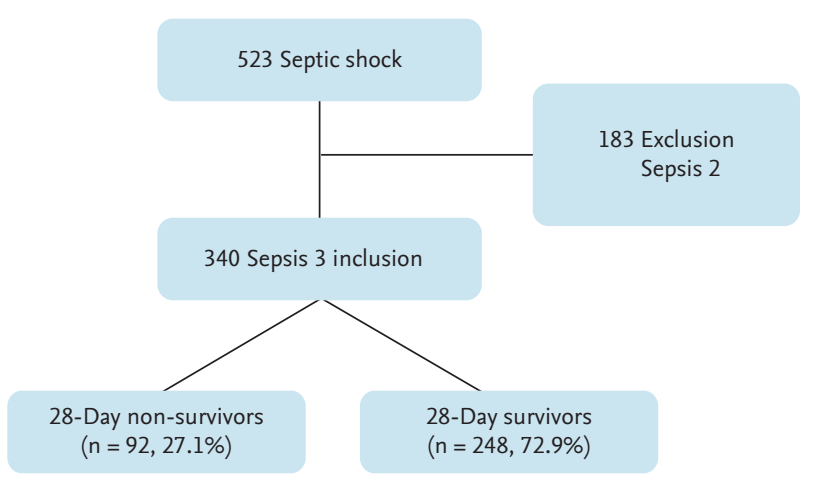

Figure 2. Flowchart of the study patients.
14), and only one patient died within 6 hours of presentation. Among the 340 patients, 92 (27.1\%) died within 28 days after presentation to the ED.

The 340 patients included 215 men (63.2\%) and 125 women (37.8\%) with a median age of 66.0 years (Table 1 ). Comorbid diseases did not differ significantly between the 28-day survivors and non-survivors. The respiratory tract $(\mathrm{n}=109,32.1 \%)$ was the most common focus of infection, followed by the hepatobiliary tract ( $\mathrm{n}=106$, $31.2 \%)$ and the urinary tract $(n=46,13.5 \%)$. Respiratory tract infection was significantly more frequent ( $44.6 \% \mathrm{vs.}$ $27.4 \%, p=0.001$ ), whereas urinary tract infection was sig-

Table 1. Demographic and clinical characteristics of the 28 -day non-survivors and survivors among patients with septic shock

\begin{tabular}{|c|c|c|c|c|}
\hline Characteristic & $\operatorname{Total}(\mathrm{n}=340)$ & 28-day non-survivor $(n=92)$ & 28 -day survivor $(n=248)$ & $p$ value \\
\hline Age, yr & $66.0(57.0-75.0)$ & $67.0(58.3-77.8)$ & $65.0(57.0-74.0)$ & 0.101 \\
\hline Male sex & $215(63.2)$ & $57(62.0)$ & $158(63.7)$ & 0.430 \\
\hline \multicolumn{5}{|l|}{ Comorbid disease } \\
\hline Hypertension & $128(37.6)$ & $30(32.6)$ & $98(39 \cdot 5)$ & 0.149 \\
\hline Diabetes mellitus & $116(34.1)$ & $32(34.8)$ & $84(33 \cdot 9)$ & 0.486 \\
\hline Liver cirrhosis & $77(22.6)$ & $21(22.8)$ & $55(22.2)$ & 0.419 \\
\hline Coronary artery disease & $37(10.9)$ & $11(12.0)$ & $26(10.5)$ & 0.415 \\
\hline Chronic kidney disease & $29(8.5)$ & $9(9.8)$ & $20(8.1)$ & 0.378 \\
\hline \multicolumn{5}{|l|}{ Focus of infection } \\
\hline Respiratory tract & $109(32.1)$ & $41(44.6)$ & $68(27.4)$ & 0.001 \\
\hline Hepatobiliary tract & $106(31.2)$ & $25(27.2)$ & $81(32.7)$ & 0.067 \\
\hline Urinary tract & $46(13.5)$ & $5(5 \cdot 4)$ & $41(16.5)$ & 0.003 \\
\hline Gastrointestinal tract & $43(12.6)$ & $8(8.7)$ & $35(14.1)$ & 0.063 \\
\hline Others & $47(13.8)$ & $12(13.0)$ & $35(14.1)$ & 0.138 \\
\hline \multicolumn{5}{|l|}{ Laboratory results } \\
\hline WBC, $\times 10^{3} / \mu \mathrm{L}$ & $10.5(4.3-17.9)$ & $9.4(3.6-16.6)$ & $10.8(4.7-28.0)$ & 0.312 \\
\hline Hemoglobin, g/dL & $10.6(9.0-12.5)$ & $10.5(8.7-12.1)$ & $10.6(9.1-12.7)$ & 0.165 \\
\hline Platelet, $\times 10^{3} / \mu \mathrm{L}$ & $134.5(63.0-196.3)$ & $130.0(45 \cdot 5-191.5)$ & $135.0(68.0-197.0)$ & 0.309 \\
\hline Creatinine, mg/dL & $1.6(1.1-2.6)$ & $1.7(1.1-2.7)$ & $1.6(1.1-2.6)$ & 0.271 \\
\hline Total bilirubin, mg/dL & $1.2(0.6-2.6)$ & $1.2(0.5-2.7)$ & $1.2(0.7-2.6)$ & 0.254 \\
\hline Prothrombin time, INR & $1.4(1.2-1.7)$ & $1.4(1.3-1.9)$ & $1.3(1.2-1.6)$ & 0.005 \\
\hline $\mathrm{CRP}, \mathrm{mg} / \mathrm{dL}$ & $12.8(4.8-22.0)$ & $15.9(6.8-26.1)$ & $11.5(4.3-21.3)$ & 0.022 \\
\hline Troponin I, ng/mL & $0.06(0.01-0.23)(n=266)$ & $0.07(0.02-0.28)(\mathrm{n}=78)$ & $0.05(0.01-0.22)(n=188)$ & 0.211 \\
\hline Lactate, mmol/L & $5.1(3.5-7.4)$ & $6.5(3.7-9.9)$ & $4.8(3.3-6.6)$ & 0.001 \\
\hline SOFA score & $6.0(4.0-8.0)$ & $7.0(5.0-9.0)$ & $6.0(4.0-8.0)$ & 0.001 \\
\hline
\end{tabular}

Values are presented as median (interquartile range) or number (\%).

WBC, white blood cell; INR, international normalized ratio; CRP, C-reactive protein; SOFA, sequential organ failure assessment. 
Table 2. Comparison of the hemodynamic parameters that affect 28 -day mortality

\begin{tabular}{|c|c|c|c|c|}
\hline TWA-6-hr & Total $(n=340)$ & Non-survivor $(n=92)$ & Survivor $(n=248)$ & $p$ value \\
\hline MAP, mmHg & $71.6(67.8-77.7)$ & $71.4(68.0-77.9)$ & $71.7(67.7-77.7)$ & 0.879 \\
\hline $\mathrm{SBP}, \mathrm{mmHg}$ & $99.2(92.3-108.0)$ & $101.5(93.2-112.7)$ & $98.4(91.6-106.7)$ & 0.076 \\
\hline $\mathrm{DBP}, \mathrm{mmHg}$ & $57.8(53.3-63.4)$ & $57.1(51.2-63.0)$ & $58.2(53.7-63.7)$ & 0.153 \\
\hline SI & $1.06(0.92-1.23)$ & $1.06(0.94-1.27)$ & $1.06(0.90-1.22)$ & 0.244 \\
\hline $\mathrm{PP}, \mathrm{mmHg}$ & $40.3(34.1-48.0)$ & $43.0(34.5-53.4)$ & $39.2(33.9-46.1)$ & 0.020 \\
\hline
\end{tabular}

TWA-6-hr, time-weighted average during the first 6 hours after septic shock; MAP, mean arterial pressure; SBP, systolic blood pressure; DBP, diastolic blood pressure; SI, shock index; PP, pulse pressure.

Table 3. Multivariable logistic regression analysis for predicting the effects on 28 -day mortality in patients with septic shock

\begin{tabular}{lccc}
\hline Variable & Odds ratio & 95\% Confidence interval & $p$ value \\
\hline Lung infection & 2.05 & $1.209-3.470$ & 0.008 \\
CRP & 1.03 & $1.004-1.049$ & 0.021 \\
Lactate & 1.14 & $1.057-1.234$ & 0.001 \\
SOFA score & 1.10 & $1.014-1.192$ & 0.022 \\
6-hour TWA pulse pressure & 1.28 & $1.012-1.622$ & 0.039 \\
\hline
\end{tabular}

CRP, C-reactive protein; SOFA, sequential organ failure assessment; TWA, time-weighted average.

nificantly less frequent (5.4\% vs. $16.5 \%, p=0.003$ ) in the 28-day non-survivors than in the survivors.

Assessments of laboratory findings revealed that prothrombin time (international normalized ratio, 1.4 vs. 1.3; $p=0.005)$, lactate concentration $(6.5 \mathrm{mmol} / \mathrm{L}$ vs. 4.8 $\mathrm{mmol} / \mathrm{L}, \mathrm{p}=0.001)$, C-reactive protein concentration $(15.9 \mathrm{mg} / \mathrm{dL}$ vs. $11.5 \mathrm{mg} / \mathrm{dL}$ ), and sequential organ failure assessment (SOFA) score (7.0 vs. 6.0, $p=0.001$ ) were significantly higher in the non-survivors than in the survivors, whereas white blood cell and platelet counts did not significantly differ between the two groups.

\section{TWA hemodynamic parameters and outcomes}

Table 2 shows the TWA hemodynamic parameters of the patients with septic shock, grouped by 28 -day survival. The median TWP-PP was significantly higher in the non-survivors (43.0 vs. 39.2, $p=0.020$ ), whereas other hemodynamic parameters, including MAP, SBP, DBP, and SI, did not significantly differ between the two groups.

The multivariable analysis revealed that 6-hour TWA PP (adjusted OR, $1.28 \mathrm{mmHg}$; 95\% CI, 1.012 to 1.622; $p=$ 0.039), lung infection (adjusted OR, 2.05; 95\% CI, 1.209 to $3.470 ; p=0.008$ ), C-reactive protein concentration (ad-

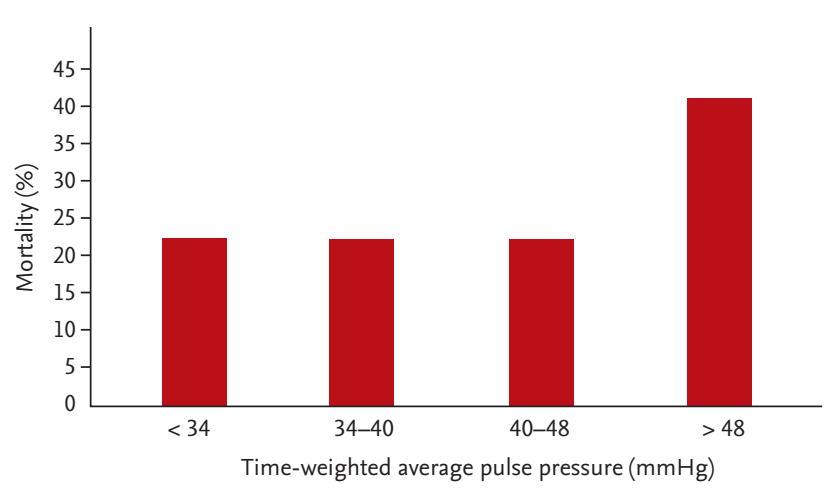

Figure 3. Relationship between 28-day mortality rate and quartile of time-weighted average pulse pressure.

justed OR, 1.03; 95\% CI, 1.004 to 1.049; $p=0.021$ ), lactate concentration (adjusted OR, 1.14; 95\% CI, 1.057 to 1.234; $p=0.001$ ), and SOFA score (adjusted OR, 1.10; 95\% CI, 1.014 to $1.192 ; p=0.022$ ) were independent predictors of 28-day mortality in patients with septic shock (Table 3).

\section{Quartiles of TWA PP and 28-day mortality}

When TWA PP was divided into quartiles ( $<34,34$ to 40 , 40 to 48 , and $>48 \mathrm{mmHg}$ ), the 28 -day mortality rates in these subgroups were $22.4 \%, 22.4 \%, 22.4 \%$, and $41.2 \%$, re- 
spectively (Fig. 3). The multivariable logistic regression analysis revealed that PPs of $>48 \mathrm{mmHg}(\mathrm{OR}, 2.25 ; 95 \%$ CI, 1.272 to $3.981 ; p=0.005$ ) were independently associated with the 28 -day mortality rate.

\section{DISCUSSION}

This study was performed to evaluate the association between hemodynamic parameters during the initial resuscitative period and 28-day mortality rates in patients with septic shock. Among the TWA SBP, DBP, MAP, SI, and PP during the first 6 hours after shock recognition, we found that only higher TWA PPs were significantly associated with patient mortality.

In our study, a high TWA PP in the early phase of septic shock showed an independent association with 28-day mortality. High PP is a strong predictor of cardiovascular morbidity/mortality in the geriatric population and among patients with hypertension, coronary heart disease, diabetes mellitus, and renal disease [16-20]. However, there is a paucity of studies about the prognostic value of $\mathrm{PP}$ in regards to mortality of patients with sepsis, especially among patients administered a resuscitation therapy bundle including maintaining $\mathrm{MAP}$ at $>65 \mathrm{mmHg}$ [21].

PP is a surrogate parameter of arterial stiffness, but it is also a dynamic parameter that is instantly influenced by the pattern of ventricular ejection, distensibility of the large arteries, the timing of reflected waves, and the HR [18]. Administration of fluids and vasopressors to increase the MAP during the early resuscitation phase would cause different changes of blood parameters according to the patient's status (i.e., an increase in SBP or DBP only or increases of both). Vasopressors and fluids can cause an increase in SBP with maintenance of DBP, and consequently, a high PP in elderly patients or others, who are highly likely to have increased arterial stiffness [22].

Lamontagne et al. [22] recently demonstrated no difference in mortality between a permissive hypotension (MAP target, 6o to $65 \mathrm{mmHg}$ ) group and a usual care group in older critically ill patients with vasodilatory hypotension. The results of this study imply that artificially raising the blood pressure by administration of high-dose catecholamine agents will cause harm and no benefit for septic shock patients, and other physiologic variables are more appropriate for targeting and monitoring [22-26]. A low PP might be one of the target physiologic variables, allowing for a MAP target of 60 to $65 \mathrm{mmHg}$, especially in elderly patients or those with preexisting hypertension.

A previous study assessing the correlation between PP and mortality in patients with sepsis found that the mortality rate was significantly lower in patients with $\mathrm{PP}>70 \mathrm{mmHg}$ than in those with lower PPs (OR, 0.67; 95\% CI, 0.54 to $0.83 ; p=0.003)$, suggesting that PP could be used as a prognostic factor to assess and implement management in patients with sepsis [21]. The discrepancy between their results and our findings may be due to the differences between the patient populations (septic shock vs. sepsis) and blood pressure measurement methods (indwelling arterial catheter vs. arm cuffs). Moreover, in contrast to measuring blood pressure parameters once, at ED admission, we evaluated patients' vital signs by continuous measures of cumulative parameters. We thought the TWA could reflect the variability of hemodynamic parameters during the resuscitation period more effectively than assessment of changes in hemodynamic parameters at regular time points. Our study design is therefore more appropriate to determine the association between PP and mortality. Another possible explanation for the inter-study discrepancy may be that septic shock in our patients was defined in accordance with the Sepsis-3 definition; thus, it included septic shock patients with more severe conditions and worse outcomes than those included in prior studies using outdated definitions of septic shock [27,28].

SI, calculated as HR divided by SBP, has been associated with early recognition and mortality of septic and hypovolemic shock [29-32]. In this study, however, the SIs in the patients with septic shock did not have a significant impact on survival outcomes.

This study had several limitations. Firstly, although all the patients were treated in accordance with the latest guidelines, information on fluids and vasopressor agents used during the acute phase of sepsis was not analyzed together, and their potential effects were not controlled. Secondly, cardiovascular function would affect the PP, which is an important confounding factor that should be adjusted. However, the majority of our study patients were not evaluated the cardiovascular function 
during the resuscitation period, and this would be considered to interpret our results. Thirdly, owing to its short follow-up period, long-term outcomes in patients with sepsis could not be determined. Finally, we did not consider the effect of vasopressor use and volume status, which could affect the hemodynamic parameters.

In conclusion, of all the hemodynamic parameters measured, only PP was significantly associated with 28-day mortality rates in patients with septic shock, defined in accordance with Sepsis-3 criteria, with the MAP maintained at $>65 \mathrm{mmHg}$ during the first 6 hours. This study, however, did not show that adding PP as a new therapeutic target could improve mortality rates.

\section{KEY MESSAGE}

1. During septic shock initial resuscitation, the target ranges of hemodynamic parameters being lack of understanding.

2. During the first 6 hours, maintaining the adequate mean arterial pressure as well as optimize pulse pressure was significantly associated with 28-day survival in patients with septic shock.

\section{Conflict of interest}

No potential conflict of interest relevant to this article was reported.

\section{REFERENCES}

1. Singer M, Deutschman CS, Seymour CW, et al. The Third International Consensus Definitions for Sepsis and Septic Shock (Sepsis-3). JAMA 2016;315:801-810.

2. Rhodes A, Evans LE, Alhazzani W, et al. Surviving Sepsis Campaign: International Guidelines for Management of Sepsis and Septic Shock: 2016. Intensive Care Med 2017;43:304-377.

3. Leone M, Asfar P, Radermacher P, Vincent JL, Martin C. Optimizing mean arterial pressure in septic shock: a critical reappraisal of the literature. Crit Care 2015;19:101.

4. Asfar P, Meziani F, Hamel JF, et al. High versus low blood-pressure target in patients with septic shock. N Engl J Med 2014;370:1583-1593.
5. Gillebert TC. Pulse pressure and blood pressure components:. Is the sum more than the parts? Eur J Prev Cardiol 2018;25:457-459.

6. Dart AM. Should pulse pressure influence prescribing? Aust Prescr 2017;40:26-29.

7. Sobiczewski W, Wirtwein M, Jarosz D, Trybala E, Bieniaszewski L, Gruchala M. Increased total mortality as a function of 24 -h pulse pressure dipping. J Hum Hypertens 2016;30:100-104.

8. Osawa T, Fujihara K, Harada M, et al. Higher pulse pressure predicts initiation of dialysis in Japanese patients with diabetes. Diabetes Metab Res Rev 2019;35:e3120.

9. Vemmos KN, Tsivgoulis G, Spengos K, et al. Pulse pressure in acute stroke is an independent predictor of longterm mortality. Cerebrovasc Dis 2004;18:30-36.

10. Shin TG, Hwang SY, Kang GH, et al. Korean Shock Society septic shock registry: a preliminary report. Clin Exp Emerg Med 2017;4:146-153.

11. Dellinger RP, Levy MM, Rhodes A, et al. Surviving sepsis campaign: international guidelines for management of severe sepsis and septic shock: 2012. Crit Care Med 2013;41:580-637.

12. Casserly B, Phillips GS, Schorr C, et al. Lactate measurements in sepsis-induced tissue hypoperfusion: results from the Surviving Sepsis Campaign database. Crit Care Med 2015;43:567-573.

13. Ryoo SM, Lee J, Lee YS, et al. Lactate level versus lactate clearance for predicting mortality in patients with septic shock defined by sepsis-3. Crit Care Med 2018;46:e489-e495.

14. Ryoo SM, Ahn R, Lee J, et al. Timing of repeated lactate measurement in patients with septic shock at the emergency department. Am J Med Sci 2018;356:97-102.

15. Kim JS, Kim YJ, Ryoo SM, et al. One-year progression and risk factors for the development of chronic kidney disease in septic shock patients with acute kidney injury: a single-centre retrospective cohort study. J Clin Med 2018;7:554.

16. Gasowski J, Fagard RH, Staessen JA, et al. Pulsatile blood pressure component as predictor of mortality in hypertension: a meta-analysis of clinical trial control groups. J Hypertens 2002;20:145-151.

17. Tadic M, Quarti-Trevano F, Bombelli M, et al. The importance of pulse pressure on cardiovascular risk and total mortality in the general population: is sex relevant? J Clin Hypertens (Greenwich) 2018;20:1001-1007. 
18. Cavalcante JL, Lima JA, Redheuil A, Al-Mallah MH. Aortic stiffness: current understanding and future directions. J Am Coll Cardiol 2011;57:1511-1522.

19. Su N, Zhai FF, Ni J, et al. Pulse pressure within 3 months after ischemic stroke is associated with long-term stroke outcomes. Am J Hypertens 2017;30:1189-1195.

20. Harbaoui B, Nanchen D, Lantelme P, et al. Prognostic value of pulse pressure after an acute coronary syndrome. Atherosclerosis 2018;277:219-226.

21. Al-Khalisy H, Nikiforov I, Jhajj M, Kodali N, Cheriyath P. A widened pulse pressure: a potential valuable prognostic indicator of mortality in patients with sepsis. J Community Hosp Intern Med Perspect 2015;5:29426.

22. Lamontagne F, Richards-Belle A, Thomas K, et al. Effect of reduced exposure to vasopressors on 9o-day mortality in older critically ill patients with vasodilatory hypotension: a randomized clinical trial. JAMA 2020;323:938-949.

23. Lamontagne F, Day AG, Meade MO, et al. Pooled analysis of higher versus lower blood pressure targets for vasopressor therapy septic and vasodilatory shock. Intensive Care Med 2018;44:12-21.

24. Takala J. Should we target blood pressure in sepsis? Crit Care Med 2010;38(10 Suppl):S613-S619.

25. Lamontagne F, Meade MO, Hebert PC, et al. Higher versus lower blood pressure targets for vasopressor therapy in shock: a multicentre pilot randomized controlled trial. Intensive Care Med 2016;42:542-550.

26. Marshall JC. Choosing the best blood pressure target for vasopressor therapy. JAMA 2020;323:931-933.

27. Ryoo SM, Kang GH, Shin TG, et al. Clinical outcome comparison of patients with septic shock defined by the new sepsis-3 criteria and by previous criteria. J Thorac Dis 2018;10:845-853.

28. Ko BS, Kim K, Choi SH, et al. Prognosis of patients excluded by the definition of septic shock based on their lactate levels after initial fluid resuscitation: a prospective multi-center observational study. Crit Care 2018;22:47.

29. Yasaka Y, Khemani RG, Markovitz BP. Is shock index associated with outcome in children with sepsis/septic shock?* Pediatr Crit Care Med 2013;14:e372-e379.

30. Berger T, Green J, Horeczko T, et al. Shock index and early recognition of sepsis in the emergency department: pilot study. West J Emerg Med 2013;14:168-174.

31. Sohn CH, Kim YJ, Seo DW, et al. Blood lactate concentration and shock index associated with massive transfusion in emergency department patients with primary postpartum haemorrhage. Br J Anaesth 2018;121:378-383.

32. Ko BS, Kim YJ, Jung DH, et al. Early risk score for predicting hypotension in normotensive patients with non-variceal upper gastrointestinal bleedin. J Clin Med 2019;8:37. 
KJIM'

Supplementary Table 1. Linear regression analysis for 28 -day survivor

\begin{tabular}{|c|c|c|c|c|c|c|}
\hline Dependent & Predictors & B & Beta & $t$ & $p$ value & VIF \\
\hline \multirow[t]{8}{*}{ 28-day survivor } & $\operatorname{Sex}$ & -0.25 & -0.027 & -0.506 & 0.613 & 1.060 \\
\hline & Age & 0.002 & 0.066 & 1.254 & 0.211 & 1.071 \\
\hline & UTI & -0.159 & -0.122 & -2.257 & 0.025 & 1.125 \\
\hline & Lung infection & 0.106 & 0.112 & 2.106 & 0.036 & 1.076 \\
\hline & CRP & 0.005 & 0.119 & 2.287 & 0.023 & 1.033 \\
\hline & Lactate & 0.026 & 0.187 & $3 \cdot 546$ & $<0.001$ & 1.059 \\
\hline & TWA PP quartile & 0.038 & 0.095 & 1.768 & 0.078 & 1.109 \\
\hline & SOFA & 0.019 & 0.133 & 2.513 & 0.012 & 1.069 \\
\hline
\end{tabular}

VIF, variance inflation factor; UTI, urinary tract infection; CRP, C-reactive protein; TWA PP, time-weighted average pulse pressure; SOFA, sequential organ failure assessment. 